\title{
Efectividad de las TIC implementadas por el CEITICB en la UNAH desde 2008 a 2013
}

Leonel Ernesto Zúniga Mendoza ${ }^{1}$

\section{RESUMEN}

La presente investigación buscó determinar cuál ha sido la efectividad de las principales tecnologías de información y comunicación (TIC) implementadas por el CEITICB desde el año 2008, tanto a nivel de los estudiantes como a nivel docente en la UNAH. Se aplicó un enfoque mixto (cuantitativo y cualitativo). Se usaron encuestas sobre el uso del software de visualización de modelos moleculares (RASMOL y JMOL) en laboratorio a un total de 328 estudiantes del área de la salud que cursaron las clases de Biología General, Biología Médica, Genética Médica y Biología General II. Se encuestaron 30 docentes de los centros regionales de la UNAH sobre el uso de la Intranet del CEITICB como medio de acceso a recursos educativos. Se realizaron 6 grupos focales para evaluar el uso de las TIC y el Proyecto Aulas Multimedia Autosuficientes en profesores y estudiantes del Departamento y Carrera de Biología. Los resultados mostraron que las TIC implementadas por el CEITICB han sido efectivas en el desarrollo de competencias en los estudiantes y profesores, favoreciendo el proceso enseñanza aprendizaje de forma diferencial. Para lograr un mayor impacto en el uso de software de visualización de modelos moleculares es necesario que los profesores integren su uso en el salón de clases. El Proyecto Aulas Multimedia Autosuficientes, mostró ser un eficiente modelo de equipamiento multimedia de aulas a nivel universitario.

Palabras claves: TIC, modelos moleculares, RASMOL, JMOL, 3D, proyección multimedia, recursos educativos.

\footnotetext{
${ }^{1}$ Beneficiario de una beca básica de la DICYP. Coordinador del Centro de Innovación en Tecnologías de Información y Comunicación para Ciencias Biológicas (CEITICB), Escuela de Biología, Facultad de Ciencias, UNAH: ceiticb@unah.edu.hn
} 


\section{ABSTRACT}

This research sought to determine what has been the effectiveness of the main Information and Communication Technologies (ICTs) implemented by the CEITICB since 2008, both at the level of students and teachers in the UNAH. A mixed approach was applied (quantitative and qualitative). Surveys were applied on the use of molecular visualization software models (Rasmol and Jmol) in laboratory to a total of 328 students that coursed the following classes: General Biology, Medical Biology, Medical Genetics and General Biology II. 30 teachers of the Regional Centers of the UNAH were surveyed on the use of the CEITICB Intranet as a means of access to educational resources. Six focus groups were performed to evaluate the use of TICs and the Self Sufficient Multimedia Classrooms project for teachers and students of the Biology department. The results showed that the ICTs implemented by the CEITICB have been effective, the students and teachers have developed competences stimulates teaching and learning process differentially. To achieve a greater impact on the use of molecular visualization software models, the teachers need to integrate the use in the classroom. The Self Sufficient Multimedia Classrooms project proved to be an efficient model of multimedia classroom equipment at university level.

Keywords: TIC, molecular models, Rasmol, Jmol 3D multimedia projection, educational resources. 


\section{INTRODUCCIÓN}

El Departamento de Biología de la UNAH ha llevado un proceso sostenido de implementación de las TIC desde el año 2003, fecha en la cual se instaló la primera red de acceso a internet en el edificio de Ciencias Biológicas, sede de este departamento. En el 2004, se instala el primer laboratorio de cómputo con acceso a internet y en el 2007, se abrió la primera sala de proyecciones multimedia en el mismo edificio, dando origen en el mismo año a la creación de la Unidad de Tecnología Educativa para las Ciencias Biológicas (UTECB), que luego pasó a denominarse Centro de Innovación en Tecnologías de Información y Comunicación para Ciencias Biológicas (CEITICB) en el 2011.

Desde el año 2008, el CEITICB ha implementado en el Departamento de Biología, varios proyectos de tecnologías de información y comunicación a nivel educativo, siendo algunos de las más importantes los siguientes:

1. Proyecto RASMOL: práctica de visualización de modelos moleculares orientada a estudiantes del área de la salud que cursan las asignaturas de Biología General y Biología Médica utilizando los softwares RASMOL y JMOL.

2. Intranet Escuela de Biología: servicio web de acceso interno en la UNAH que permite a los centros regionales de la UNAH acceder a recursos educativos utilizados en el Departamento de Biología en Ciudad Universitaria.

3. TIC implementadas en la asignatura Biología General II: la asignatura de Biología General II ha brindado el espacio para hacer pruebas piloto de varias tecnologías que posteriormente han sido implementadas en otras asignaturas del Departamento de Biología.

4. Proyecto Aulas Multimedia Autosuficientes: un modelo de equipamiento multimedia de aulas implementado en el Departamento de Biología.

Caber resaltar, que el proceso de Reforma en la UNAH y la actual administración favorecieron, directa e indirectamente, el avance de las TIC que ha tenido el Departamento de Biología gracias al funcionamiento del CEITICB.

Sin embargo, hay un desconocimiento de la efectividad que han tenido las TIC implementadas por el CEITICB en la UNAH desde el año 2008 a 2013, tanto a nivel de los 
profesores como a nivel de los estudiantes en competencias y conocimientos adquiridos. Asimismo, se desconoce cuál ha sido la efectividad en los estudiantes de las carreras del área de la salud que cursan clases impartidas por la Escuela de Biología y en los estudiantes de los centros regionales de la UNAH.

\section{METODOLOGÍA}

En la realización de esta investigación se utilizó un enfoque mixto (cuantitativo y cualitativo) y se realizó durante el segundo periodo académico de 2014. El segmento cuantitativo de la presente investigación es no experimental, ya que las tecnologías a ser evaluadas se utilizan periódicamente en la UNAH desde el 2008.

El segmento cualitativo utilizado en la investigación es de tipo narrativo, ya que pretende recolectar datos de la experiencia de estudiantes y profesores de la Escuela de Biología, con el uso de las TIC, para describirla y analizarla. Es de tipo transversal, puesto que la recolección de datos será en un momento único del tiempo.

Para medir la efectividad de RASMOL y JMOL se diseñaron pruebas escritas con imágenes de los modelos moleculares observados en las prácticas de estos (instrumentos II-1, II-2, II-3, II-4) y se aplicaron a estudiantes de carreras del área de la salud que cursaban la asignatura de Biología General, de la Carrera de Medicina y Nutrición que llevaban la materia de Biología Médica y de la carrera de Medicina en la clase de Genética Médica. Para medir la efectividad de la Intranet de la Escuela de Biología, se diseñó una encuesta electrónica que se aplicó a los profesores de los centros regionales de la UNAH que impartieron las asignaturas de Biología General, Biología Médica y Educación Ambiental, durante el segundo periodo académico de 2014.

Para determinar cualitativamente la efectividad de las TIC implementadas en la asignatura de Biología General II, se realizaron tres grupos focales durante el primer y segundo periodo académico de la UNAH del año 2014. Para determinar cualitativamente la efectividad del Proyecto Aulas Multimedia Autosuficientes (Proyecto AMA) se realizaron tres grupos focales con estudiantes y profesores. 


\section{RESULTADOS}

1. Efectividad de los softwares RASMOL y JMOL, nivel de identificación

Los instrumentos de investigación se aplicaron a un total de 432 estudiantes (238 de Biología General y Biología Médica, 148 de Genética Médica y 46 de Biología General II), una semana después de la finalización del desarrollo de las prácticas en evaluación.

Del grupo a analizar se aislaron los estudiantes que cursaban dichas asignaturas por segunda vez, por lo que la población se redujo a 328 estudiantes, de los cuales 79 no realizaron las práctica de RASMOL y 249 si realizaron las prácticas RASMOL (99) y JMOL (150).

Se determinó por estudiante, el porcentaje de identificación de estructuras moleculares presentadas en los instrumentos II-1 para la práctica RASMOL para los estudiantes de Biología General BI-121 y Biología Médica BI-123, instrumentos II-2 y II-3 para las prácticas JMOL1 y JMOL2 para Genética Médica y el instrumento II-4 para la práctica JMOL 3D para los estudiantes de Biología General II.

Debido a que las prácticas RASMOL para Biología General BI-121 y Biología Médica BI-123, JMOL1 y JMOL2 para Genética Médica y JMOL 3D para los estudiantes de Biología General II son diferentes, se determinó el porcentaje de estructuras que cada estudiante logró identificar de los instrumentos aplicados para poder integrar los datos en un solo análisis.

Para el análisis de los porcentajes de identificación se establecieron 9 niveles con base al porcentaje de estructuras identificadas (ver cuadro 1 y gráfico 1):

$\begin{array}{lll}1=0 \% & 4=31-50 & 7=71-80 \\ 2=1-20 & 5=51-60 & 8=81-90 \\ 3=21-30 & 6=61-70 & 9=91-100\end{array}$


Cuadro 1. Distribución porcentual de los estudiantes según la práctica evaluada con base en los niveles de identificación, mediante aplicación de los instrumentos II-1, II-2, I-3 y II-4

\begin{tabular}{|c|c|c|c|c|c|c|c|c|c|c|}
\hline \multicolumn{11}{|c|}{ Niveles de identificación } \\
\hline & N1 & N2 & N3 & N4 & N5 & N6 & N7 & N8 & N9 & \\
\hline RASMOL no* & 33 & 35 & 13 & 9 & 5 & 0 & 5 & 0 & 0 & 100 \\
\hline RASMOL sí* & 8 & 17 & 20 & 12 & 22 & 0 & 15 & 8 & 17 & 100 \\
\hline JMOL1, 2 & 4 & 21 & 17 & 24 & 9 & 4 & 8 & 6 & 7 & 100 \\
\hline JMOL 3D & 12 & 18 & 0 & 15 & 6 & 30 & 0 & 0 & 18 & 100 \\
\hline
\end{tabular}

Gráfico 1. Comparación del porcentaje de estudiantes de las clases Biología General y Biología Médica que identificaron modelos moleculares específicos mostrados en el instrumento II-1, una semana después del abordaje del tema de composición Qu

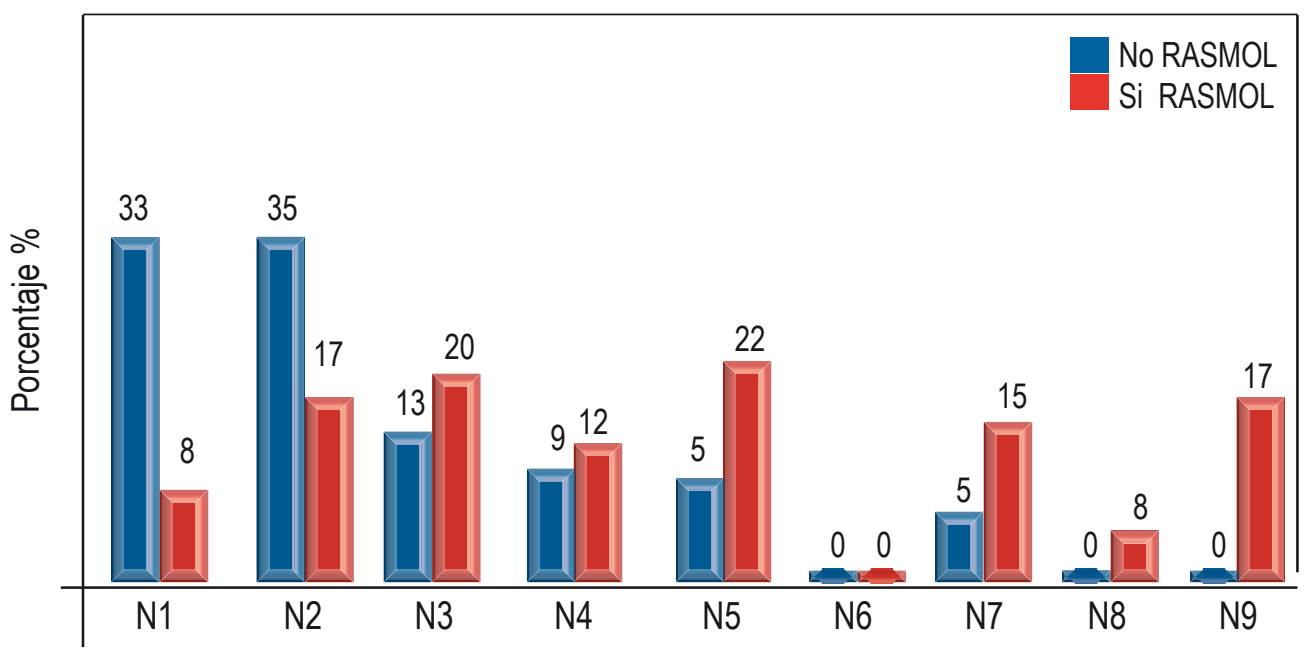

Como puede observarse en el gráfico 1, de los estudiantes que no realizaron la práctica RASMOL, el $33 \%$ no logró identificar ninguna estructura y el $35 \%$ de los estudiantes alcanzaron únicamente el nivel 2; lo que implica que el $68 \%$ de estos estudiantes mostró una pobre competencia para identificar modelos moleculares. El $57 \%$ de los estudiantes $(35+13+9)$ no logró identificar más del $50 \%$ de las estructuras mostradas en el instrumento. Un $10 \%(5+0+5+0+0)$ logró identificar más de la 
mitad de los modelos moleculares y, de este grupo, ningún estudiante mostró una alta competencia para identificar modelos moleculares (niveles 8 y 9 ).

En contraste, en el caso de los estudiantes que sí realizaron la práctica RASMOL, solo el $8 \%$ no logró identificar ninguna estructura y el $17 \%$ de los estudiantes alcanzaron únicamente el nivel 2; lo que significa que solo el $25 \%$ de estos estudiantes tiene una pobre competencia para identificar modelos moleculares. El 49 $\%$ de los estudiantes $(17+20+12)$ no logró reconocer más del $50 \%$ de estructuras mostradas en el instrumento. Un $42 \%(22+0+15+4+1)$ pudo señalar más de la mitad de los modelos moleculares y, de este grupo, $5 \%(4+1)$ tuvo una alta competencia para identificar modelos moleculares (niveles 8 y 9 ).

2. Efectividad de los softwares RASMOL y JMOL, identificación específica y relación

Con los datos obtenidos de la aplicación del instrumento II-1 en la evaluación de RASMOL, también se determinó el porcentaje de estudiantes que logró identificar modelos de moléculas específicas, las partes de dichas moléculas, la relación de las moléculas con temas abordados en sus clases y su apreciación personal del beneficio de la realización de la práctica; tanto para el grupo que sí realizó la práctica, como para el que no la hizo.

Gráfico 2. Comparación del porcentaje de estudiantes de las clases Biología General y Biología Médica que identificaron modelos moleculares específicos, mostrados en el instrumento II-1, una semana después del abordaje del tema de composición Qu

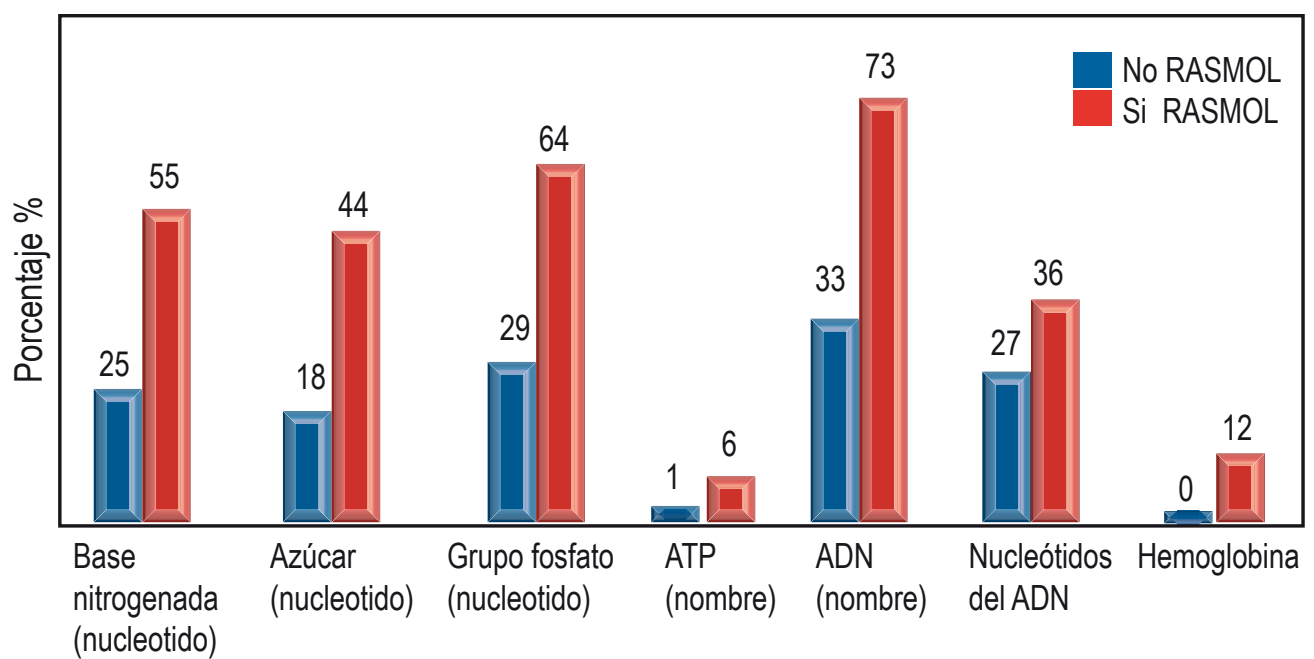


En el gráfico 2 se nota que el grupo de los estudiantes que sí desarrolló la práctica RASMOL, logró porcentajes más altos de identificación de modelos moleculares específicos, en comparación con el grupo de estudiantes que no desarrolló la práctica. De hecho, en la mayoría de los ítems puede notarse una relación de 2:1 o mayores, excepto en la identificación de los nucleótidos como subunidades del ADN. La identificación del modelo del compuesto conocido como ATP, fue baja en ambos grupos, aunque siempre mayor en el grupo de los estudiantes que sí desarrollaron la práctica. La identificación del modelo molecular de la hemoglobina únicamente ocurrió en el grupo de los estudiantes que sí desarrollaron la práctica ( $12 \%$ contra un $0 \%)$.

En el gráfico 3 se presentan los resultados de los incisos del instrumento II-1 orientados a medir en los estudiantes si la práctica con el software RASMOL favorecía su capacidad de relacionar una molécula biológica específica con temas específicos de la clase.

Gráfico 3. Comparación del porcentaje de estudiantes de las clases Biología General y Biología Médica que relacionó correctamente los modelos moleculares de ATP y hemoglobina mostrados en el instrumento II-1 con temas específicos de teoría.

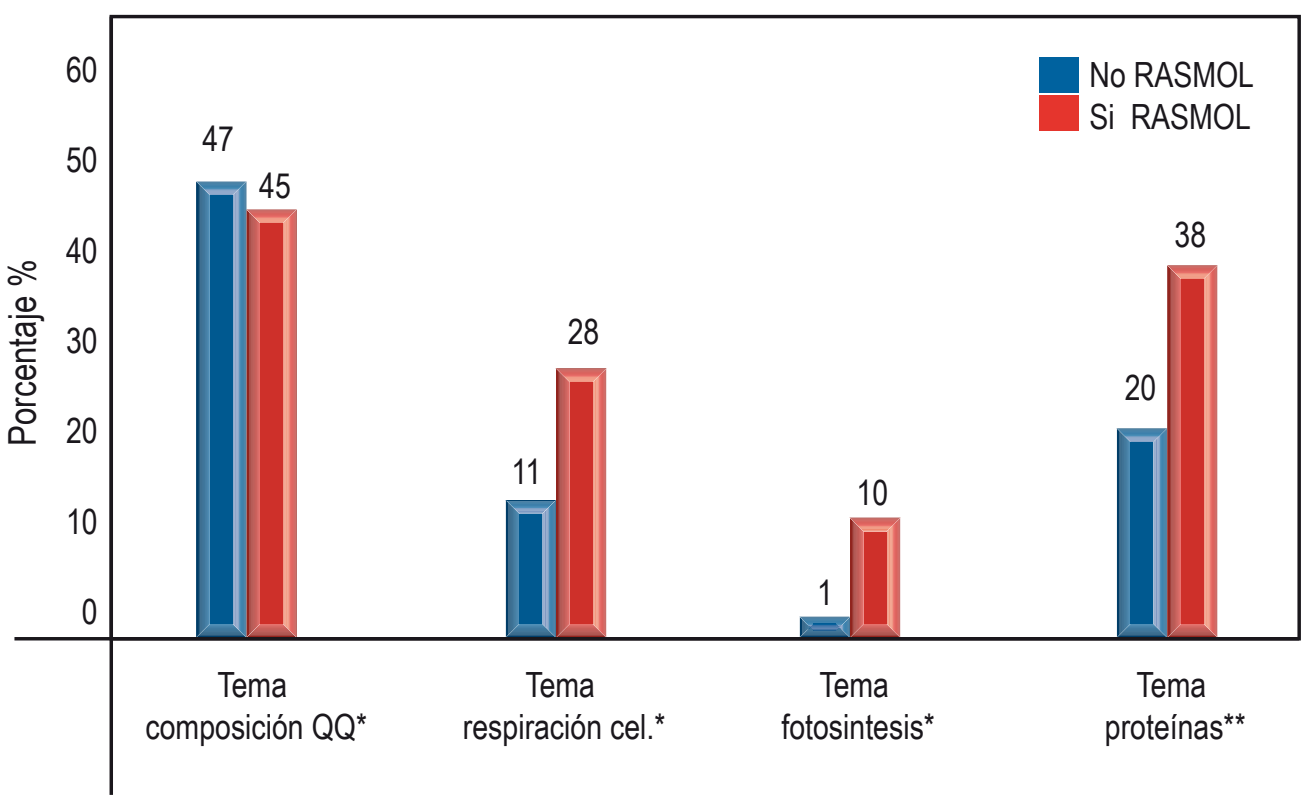


En el gráfico 3 también se puede observar que al relacionar la molécula de ATP con el tema composición química del protoplasma abordado en la clase, ambos grupos mostraron casi el mismo nivel de establecimiento de relación. De hecho, el grupo que no realizó la práctica mostró un nivel levemente mayor (47\% contra $45 \%$ ). Sin embargo, los estudiantes que desarrollaron la práctica mostraron porcentajes más altos en establecer la relación entre el ATP y los temas de respiración celular y fotosíntesis en una relación de 3:1 y 10:1, respectivamente. Es particularmente interesante este resultado, ya que estos temas no habían sido abordados en las clases teóricas para cuando se aplicó el instrumento.

En cuanto a relacionar el modelo molecular de hemoglobina con el subtema de las proteínas del tema "composición química del protoplasma", la relación es 2:1. Esta pregunta estaba directamente relacionada con la identificación de la hemoglobina (ver gráfico 2), por lo que llama la atención el hecho que fue mayor la identificación del modelo molecular de la hemoglobina como parte del tema de las proteínas en ambos grupos (ver gráfico 3), que recordar su nombre específico (ver gráfico 2).

\section{Efectividad de los softwares RASMOL y JMOL, apreciación del estudiante}

La apreciación de los estudiantes en cuanto al aporte de la práctica de RASMOL para comprender la estructura de las moléculas biológicas, mostró que un $76 \%$ de los encuestados reconoció que el desarrollo de la práctica con el software RASMOL benefició su comprensión de la estructura de las moléculas biológicas, aunque el mayor porcentaje (62 \%) considera que la misma le "ayudó poco". Un factor que puede haber influido en esta valoración es que la redacción de la segunda opción (me ayudó poco), no representa un real intermedio en las opciones de valoración, ya que la misma tiende a ser más asociada a un bajo nivel más que a un nivel intermedio. Esto no se notó en la validación del instrumento II-1, sino hasta después de su aplicación, pero se tomó en cuenta en los instrumentos II-2 y II-3 para el software JMOL. Un quinto de los encuestados $(21 \%)$ consideró que la práctica no mejoró su comprensión de la estructura de las moléculas biológicas, lo cual siempre es representativamente importante.

Para los estudiantes de Medicina,acerca de su valoración sobre la clase Genética Médica, en cuanto al aporte de las prácticas de JMOL 1 y JMOL 2 para comprender la estructura de las moléculas biológicas involucradas en el proceso genético, mostró que un $81 \%$ de los encuestados reconoció que el desarrollo de la práctica JMOL1 benefició su comprensión de la estructura de las moléculas biológicas, distribuido en un $54 \%$ que califica como de "poca ayuda", un $25 \%$ que considera que la práctica 
"mejoró" su comprensión y un 2 \% que considera que posee una comprensión superior de las estructuras moleculares estudiadas en genética. Aproximadamente un quinto de los encuestados (19\%) consideró que la práctica, no mejoró su comprensión de la estructura de las moléculas biológicas, lo cual siempre es representativamente importante. Las valoraciones de la práctica JMOL 2 es muy similar a la práctica JMOL 1.

La apreciación de los estudiantes en cuanto al aporte de la práctica de JMOL 3D para comprender la estructura de las moléculas mostró que un $91 \%$ de los encuestados reconoció que el desarrollo de la práctica JMOL 3D benefició su comprensión de la estructura de las moléculas biológicas, distribuido en un $61 \%$ que califica como de "poca ayuda" y un $30 \%$ que considera que posee una comprensión superior de las estructuras moleculares estudiadas. Solo el $9 \%$ de los encuestados consideró que la práctica no mejoró su comprensión.

4. Efectividad de la intranet de la Escuela de Biología como servicio web interno de recursos educativos a nivel de los centros regionales de la UNAH

De los 48 docentes identificados en los 9 centros regionales de la UNAH que impartieron la clase de Biología General, Biología Médica y Educación Ambiental, se logró encuestar a 30 docentes (UNAH-Valle de Sula: 8, CURC: 3, CUROC: 3, CURNO: 4, CURLP: 1, CURVA: 2, CURLA: 7, UNAH Tec-Danlí: 1 y ITST-Tela: 1).

De los docentes encuestados, solo el 30 \% confirmó haber participado en el curso: Uso de las TIC para la enseñanza de ciencias biológicas, llevado a cabo en julio del año 2012 por el CEITICB. En este curso no participó ningún profesor del ITST de Tela, ya que para entonces no había entrado en funcionamiento formalmente.

El $63 \%$ de los profesores confirmó tener conocimiento de la existencia de la intranet Escuela de Biología como recurso educativo TIC. El $43 \%$ de los encuestados confirmó haber ingresado anteriormente a la intranet Escuela de Biología de la UNAH en su propio centro regional. Solo un poco más de un tercio de ellos $(37 \%)$, únicamente cinco de los centros regionales, afirmó haber descargado recursos de esta intranet. Sin embargo, el 50 \% afirmó haber usado alguno de los recursos disponibles en la intranet con los estudiantes de las asignaturas que imparte.

En cuanto a los recursos descargados de la intranet de la Escuela de Biología por los profesores, el $37 \%$ afirmó haber descargado videos, un $17 \%$ instaladores de programas especializados, un $30 \%$ instaladores de programas comunes, un $23 \%$ 
archivos PBD de modelos moleculares para RASMOL, un $13 \%$ formatos de notas automatizados para Excel y un $7 \%$ archivos KML para Google Earth.

En cuanto a la pregunta: marque cuál de las TIC (de la intranet de la Escuela de Biología) ha implementado en su centro regional con los estudiantes de las asignaturas que imparte, se identificó que a excepción del ITST de Tela, en todos los centros regionales han descargado al menos un recurso TIC de la intranet.

La cuarta sección de la encuesta implicó preguntas abiertas como: cuántos estudiantes por periodo son beneficiarios de las TIC de la Intranet Escuela de Biología implementadas por su persona y comentarios y observaciones. Las respuestas a la primera pregunta dieron como resultado un rango de 519 a 604 estudiantes a nivel de todos los centros regionales beneficiados por las TICs provistas mediante la intranet.

5. Efectividad de las TICs implementadas en la clase de Biología II

Después de un proceso de reducción y triangulación de la transcripción de los grupos focales se obtuvieron los siguientes resultados:

a. Categoría campus virtual: sirve como un canal eficaz para la entrega de materiales de estudio al estudiante, accesible en cualquier momento, siendo a la vez una base de datos, ya que si el estudiante extraviaba los archivos de estudio, solo tenía que volver a ingresar a este servicio. Gracias a la planificación de la clase, los estudiantes pueden orientarse continuamente en los diferentes trabajos y actividades de la misma. Asimismo permite que el maestro pueda dar en cualquier momento contenido adicional al estudiante, el cual aunque no necesariamente puede ser evaluado, servía para enriquecer su clase. Reduce los costos al estudiante que posee medios para visualizar las presentaciones de la clase, ya que evita el costo de imprimirlas.

b. Categoría animaciones multimedia 2D y 3D: las demostraciones de procesos mediante estas animaciones ayudan al estudiante a aclarar y comprender mejor los procesos celulares y moleculares. Para los estudiantes la explicación no es suficiente.

c. Categoría práctica JMOL 3D: ver modelos moleculares en esta opción les permite a los estudiantes, comprender mejor la estructura tridimensional y las partes 
que componen la molécula estudiada, crea un clima atractivo de aprendizaje. La práctica JMOL 3D, puede ayudar a orientar a un estudiante hacia el estudio de biología molecular, ya que le ayuda a integrar el conocimiento del funcionamiento de una molécula con su estructura. Es una herramienta de enseñanza útil si finalmente se desempeñan como profesores después de graduarse.

d. Categoría Programa Visible Body y el IPAD: el software le permitió al estudiante tener una mayor comprensión espacial de los órganos del cuerpo humano. Con software como este es más fácil para los estudiantes establecer comparaciones con la anatomía de otros animales y les permitió exponerse a tecnologías de punta en el estudio anatómico de seres vivos.

e. Categoría Biblioteca Virtual: facilita a los estudiantes acceder a información confiable y selecta. Es una herramienta útil no solo para la clase en curso, sino también para el resto de las clases que el estudiante cursa o cursará. Las búsquedas de artículos en las bibliotecas virtuales les permitirán encontrar investigaciones interesantes que despierten su interés por leerlas, aunque no necesariamente estuvieran relacionadas con su tema de investigación, esto le da un gran valor al uso de la tecnología para el estudio e investigación. Otra ventaja es poder acceder a esta biblioteca en cualquier momento durante el día o la noche, incluso en la casa si se dispone de internet. Los estudiantes pueden realizar investigaciones bibliográficas con mucha facilidad y los capacita para saber cómo mantenerse actualizados después de finalizar su carrera.

6. Efectividad del Proyecto Aulas Multimedia Autosuficientes (Proyecto AMA): después de un proceso de reducción y triangulación de la transcripción de los grupos focales se obtuvieron los siguientes resultados:

a. Categoría que representa el Proyecto AMA para los profesores y estudiantes: para los profesores contar con este Proyecto, ha representado proveer a los estudiantes, desde el primer día de clases, un ambiente favorable para el aprendizaje, en una generación muy vinculada con la tecnología. Los estudiantes confirman esta actitud hacia la clase cuando el profesor dispone y usa el equipamiento multimedia en el aula, mostrando mayor interés cuando el profesor recurre a ayudas audiovisuales más que simplemente dictar y escribir en el pizarrón. Tanto el profesor como el estudiante valoran el hecho de no perder tiempo de clase en instalar un equipo portátil cada día y aprovechar al $100 \%$ de la hora clase. El equipamiento multimedia reduce la improvisación y permite 
compartir fácilmente recursos educativos multimedia entre profesores que imparten las mismas clases en la misma aula. El profesor puede explicar un concepto complejo con mayor rapidez al poder mostrar la ayuda visual apropiada, ya sea video, animación o imagen fija, cuando en el pasado el profesor debía invertir excesivo tiempo en dibujar un esquema en la pizarra, lo cual podía ser eficaz dependiendo de las habilidades artísticas del mismo profesor. Lo anterior también se aplica en clases como Educación Ambiental, en donde la creación de conciencia es un objetivo importante y el fácil acceso a proyección posibilita exponer a los estudiantes a situaciones reales que influyen en un cambio actitudinal. La disponibilidad de equipo multimedia permanente en las aulas favorece la actualización en TIC del profesor.

Los docentes utilizan el proyecto AMA para mostrar presentaciones de Power Point, videos, animaciones 2D y 3D, imágenes, páginas web educativas, artículos de la Biblioteca Virtual UNAH, archivos PDF, videos en Youtube, búsquedas inmediatas de recursos que aclaren un concepto a los estudiantes.

Para los profesores ser trasladados a un edificio sin equipamiento multimedia implicaría costos personales en la compra y mantenimiento de un proyector multimedia para mantener la calidad de su educación, con todos los inconvenientes implicados. La opción de impartir clases sin equipo multimedia, únicamente con pizarrón y marcador, representa para los docentes una evidente limitante para lograr cubrir con eficiencia los contenidos durante el periodo trimestral y a la vez descenso en la calidad de la enseñanza brindada, así como un menor interés del estudiante durante la clase.

Los estudiantes expresan que provocaría desmotivación en ellos, ya que por la orientación de la biología como ciencia, la visualización de imágenes del contenido estudiado es de vital importancia tanto para su estudio como para su competencia en el ejercicio profesional. Los estudiantes también expresaron la importancia para ellos del recurso multimedia como ayuda memoria para el docente, evitando explicaciones erradas. Algunos profesores expresaron también la importancia de tener cuidado de una sobredependencia de la tecnología en la educación.

b. Categoría actitud hacia la enseñanza y el aprendizaje: los profesores consideran que el uso de TIC en sus clases influye en una alta valoración de sus clases en las evaluaciones realizadas por los estudiantes. Estos también expresaron su actitud motivada cuando el profesor hace uso de recursos multimedia en el aula 
de clases, así como prestar más atención en las explicaciones al no tener que concentrarse en copiar todo lo que el profesor explica, si este facilita las presentaciones a los estudiantes. Tanto para los profesores como para los estudiantes, los recursos multimedia logran una mayor comprensión de procesos y estructuras macro y microscópicas, así como el uso de imágenes en estudios de casos, más que una simple explicación con marcador. Algunos estudiantes pueden buscar en internet los recursos para entender conceptos que no se logró comprender en la clase presencial.

c. Categoría funcionalidad del Proyecto AMA: para los profesores poder mostrar videos en el aula sin tener que visitar un salón de proyecciones es una gran utilidad del Proyecto AMA, ya que asegura la asistencia de los estudiantes durante su presentación, permite una presentación contextualizada, le evita al profesor los inconvenientes de la logística e imprevistos al utilizar un salón de proyecciones de poca disponibilidad de horarios. Para los estudiantes elimina los inconvenientes de trasladarse a otra aula o edificio; estos consideran la presentación de videos un valioso factor de motivación en una clase presencial, especialmente en horarios en los que prestar atención se les dificulta más. Igualmente, es una gran ventaja para el profesor poder mantener almacenados los videos en la computadora, ya que agiliza su presentación a los estudiantes, evita las pérdidas de tiempo y reduce los riesgos de fallas técnicas. También mejora el intercambio de recursos educativos entre profesores.

d. Categoría accesibilidad a internet en el aula de clase: permite al profesor acceder e involucrar al estudiante fácilmente con la Biblioteca Virtual. También le permite dinamismo si durante la clase percibe la necesidad de mayor claridad en una explicación o reforzarla, lo puede hacer en la misma buscando y mostrando una imagen 0 un artículo de internet. Provee una herramienta eficaz para que el profesor vincule al estudiante con un recurso web al mostrárselo durante la clase, cuando son usuarios del campus virtual pueden acceder a internet en el aula, lo cual facilita su labor docente, ya que en caso de rotación de aula solo acceden a la base de datos del campus y descargan las presentaciones para sus respectivas clases. Los estudiantes pueden revisar con el profesor durante la clase fechas e instrucciones de tareas pendientes. 


\section{ANÁLISIS DE DATOS E INFORMACIÓN}

\section{Efectividad de los softwares RASMOL y JMOL}

Comparando tanto los datos integrados como los de cada grupo de estudiantes que realizaron las prácticas RASMOL, JMOL Y JMOL 3D, se nota que las evaluaciones muestran que un poco más de un tercio de los estudiantes logró identificar más de la mitad de los modelos moleculares presentados y sus partes, una semana después de realizar dichas prácticas. En contraste, tomando como ejemplo los resultados del grupo de estudiantes que no realizó la práctica RASMOL, en el cual solo el $10 \%$ de los estudiantes reconoció más de la mitad de los modelos moleculares presentados (ver gráfico 1), queda en evidencia el beneficio educativo de realizar esta práctica con los estudiantes; asimismo muestra una fuerte evidencia del beneficio del desarrollo de las otras prácticas como JMOL y JMOL 3D.

En este punto, es importante señalar que el libro de texto utilizado en las clases Biología General y Biología Médica a la fecha del estudio, no incluían modelos moleculares a colores de los compuestos estudiados, lo cual puede ser un factor influyente en el nivel de reconocimiento de las estructuras moleculares biológicas.

Como lo expresa Stueker y otros (2003) y Muzyka (2008), el uso de modelos de moléculas generados por computadora proporcionan una mayor comprensión de los compuestos orgánicos. Esto implica que los estudiantes que realizan la práctica RASMOL podrían tener una ventaja significativa al llevar asignaturas como Química Orgánica y Bioquímica; en el caso de los estudiantes que realizan las prácticas JMOL y JMOL 3D, es una ventaja en las asignaturas de sus cursos subsiguientes que impliquen el estudio de moléculas relacionadas.

Lo anterior concuerda con los datos obtenidos por ítem de cada instrumento de evaluación. En el caso de la evaluación de RASMOL, el grupo de estudiantes que sí realizó esta práctica, mostró el doble o más del porcentaje de identificación de cada modelo molecular individual o sus partes, en comparación con el grupo que no realizó la práctica (ver gráfico 3). Esto es importante, ya que en casos como entender las partes de un nucleótido está directamente relacionado con la comprensión de temas importantes como la dinámica energética del ATP o la estructura del ADN; o como en el caso de la estructura de un aminoácido, cuyo nombre genérico implica sus partes básicas (ver gráfico 2). 
Llama la atención que los estudiantes que sí realizaron la práctica RASMOL, lograron establecer una mayor relación de los modelos moleculares con temas de la teoría estudiada en clase, aun de temas no abordados a la fecha de la aplicación del instrumento de evaluación (ver gráfico 3).

A pesar de los datos comentados anteriormente, un quinto de los estudiantes que realizaron estas prácticas calificaron su desarrollo implicando que no habían mejorado su comprensión de las moléculas biológicas y solo en el caso de la práctica JMOL $3 \mathrm{D}$ se reflejó un valor menor (9\%). Para discriminar el origen de esta apreciación se aislaron los datos de los estudiantes que expresaron esta opinión de la práctica RASMOL y resultó que el $70 \%$ de ellos fueron estudiantes que no sobrepasaron el nivel 4 de identificación, es decir, no identificaron más del $50 \%$ de las estructuras mostradas; igualmente, el $23 \%$ no sobrepasó el nivel 6 de identificación, o sea, no identificaron más del $60 \%$ de las estructuras mostradas, por lo que puede concluirse que esta apreciación en el caso de la práctica RASMOL, procede de estudiantes con un bajo rendimiento en la identificación de modelos de moléculas. Para JMOL, los valores son muy similares.

Lo expresado anteriormente también implica considerar las limitaciones de los estudiantes en habilidades tecnológicas y el nivel de complejidad de las guías desarrolladas, ya que como lo expresa Thomas y Williamson (2005), la sencillez y la interactividad son características importantes en la visualización molecular.

También es importante resaltar el papel que puede desempeñar el uso que el mismo profesor haga de estos softwares de forma personal y en el aula de clases, lo que hasta la fecha del estudio no es obligatorio ni común entre los profesores que imparten estas asignaturas. Como lo expresa Williamson y Thomas (2008), el uso de la visualización molecular eleva las habilidades espaciales de los profesores y disminuye con la falta o carencia de uso.

La integración de la visualización molecular por el profesor en la clase magistral y como una práctica de laboratorio es necesaria y no mutuamente excluyente. Lo anterior fue demostrado por el estudio de White y otros (2002), el cual compara los resultados en el aprendizaje de los estudiantes mediante una conferencia y una práctica de laboratorio en las que se utilizó la visualización de modelos moleculares, con lo cual se evidenciaron los efectos diferentes y en gran parte no traslapados, lo que sugiere que el uso efectivo de visualización molecular debe involucrar ambos tipos de actividades. 
2. Efectividad de la Intranet de la Escuela de Biología como servicio web de recursos educativos a nivel de los centros regionales de la UNAH

Aunque el $63 \%$ de los profesores encuestados manifestó tener conocimiento de la Intranet Escuela de Biología, solo el 37 \% confirmó haber descargado recursos desde este servicio web. Se destaca que un $50 \%$ de los encuestados confirmó haber utilizado recursos como los disponibles en la Intranet, lo que podría implicar que otros docentes han compartido con ellos estos recursos, o ellos mismos los han descargado de otros sitios desde el internet. Sin embargo, docentes de 7 de los 9 centros regionales confirmaron haber descargado recursos TIC de la Intranet.

Considerando las respuestas a la pregunta abierta de la encuesta (observaciones y comentarios) es probable que las limitaciones en el acceso a equipo de cómputo conectado a la plataforma tecnológica de la UNAH, las velocidades de transferencia, así como aspectos técnicos de descargas masivas, sean dificultades para que los profesores hagan uso de los recursos disponibles en la Intranet en sus centros regionales.

Otro aspecto que fue evidenciado durante el proceso de aplicación de las encuestas es que algunos de los profesores que fueron capacitados en el 2012 para el uso de la intranet de la Escuela de Biología, ya no estaban asignados a las asignaturas en estudio, factor que implica un menor uso de este recurso en el centro regional, si no hay un proceso de transferencia y capacitación entre profesores. También, este aspecto debe ser considerado por el planificador académico, al rotar el personal en diferentes asignaturas.

Al menos 519 estudiantes por periodo de 7 centros regionales diferentes (CURLA, CURLP, CURNO, CUROC, CURVA, Tec-Danlí y UNAH-VS) son beneficiarios de las TIC de la Intranet Escuela de Biología que han sido implementadas por profesores, lo que permite concluir que a pesar de las limitaciones en cuanto a la representatividad, la Intranet Escuela de Biología ha sido un medio eficiente para la trasferencia de TIC.

3. Competencias adquiridas por los estudiantes de la asignatura de Biología II por las TIC implementadas en la clase

Es notorio que en todas las TIC evaluadas de la asignatura Biología II, la interactividad de estos recursos crea un clima amigable en los estudiantes. Estos valoran mucho que el profesor utilice recursos multimedia que faciliten su proceso de gestión educativa (como el campus virtual) y la comprensión de temas difíciles (animaciones 
2D y 3D) que los puedan orientar en su estudio futuro (JMOL 3D), que los capacite para las asignaturas siguientes, en su desempeño profesional (Programa Visible Body) y que les permita cumplir con asignaciones de otras asignaturas (Biblioteca Virtual).

4. Actitudes de los profesores y estudiantes favorables a la enseñanza y aprendizaje gracias al Proyecto Aulas Multimedia Autosuficientes (AMA)

Como lo expresa el educador, escritor y conferencista británico Ken Robinson (2010), vivimos en una época en la cual, los jóvenes se exponen a la mayor estimulación intensiva de la historia, por su exposición a tantos medios poderosos de información multimedia de forma simultánea, con la cual debe competir la educación formal. De cierta manera, la afirmación anterior concuerda con lo evidenciado en los grupos focales, en donde los profesores percibían una actitud favorable de los estudiantes hacia el aprendizaje al utilizar recursos multimedia desde el primer día de clases, lo que también fue confirmado por los estudiantes. Es notorio que los estudiantes tienden a valorar más que los mismos profesores el uso de video y animaciones para comprender temas específicos de sus clases; de hecho, estos buscan resolver sus falencias en la comprensión de un tema buscando ellos mismo recursos en internet.

Para los profesores contar con el equipamiento multimedia permanente en el aula constituye un factor de seguridad en su labor académica. Movilizar un equipo diariamente e instalarlo es considerado una fuente de inconvenientes en la labor académica y su total carencia como un retroceso enorme en la calidad por él brindada. En este punto, es importante discriminar el hecho de que esta evaluación ha sido aplicada en el área de las ciencias biológicas, que por su orientación como ciencia y su objeto de estudio, los seres vivos, requiere de un recurso visual abundante para su enseñanza; por lo que esta apreciación no necesariamente puede ser aplicada en su totalidad a todas las áreas de la ciencia.

La característica del Proyecto AMA de permitir almacenar los videos y las presentaciones, presentar videos con audio y poder ingresar a internet, lo vuelven profundamente eficaz para darle dinamismo a las clases, reduciendo los problemas por fallas técnicas y asegurando un uso eficiente de la hora-clase cada día. 


\section{CONCLUSIONES}

1. Los softwares RASMOL y JMOL han mostrado ser recursos educativos eficaces, mejorando la capacidad de los estudiantes del área de la salud de la UNAH de comprender la estructura de las moléculas biológicas, pero es necesario contrastar esta información con la integración que el profesor haga de estas herramientas en su salón de clases.

2. La intranet Escuela de Biología, en cuanto a la representatividad de los centros regionales de la UNAH, puede ser considerado como un recurso eficaz para las distribución nacional de recursos TIC, sin embargo, su efectividad ha sido baja en cuanto a los estudiantes beneficiarios de estas tecnologías considerando la matrícula total por periodo, lo que se debe en parte a las limitantes de los centros regionales en cuanto a la accesibilidad que se le brinda a los profesores a equipo de cómputo e internet, la falta de asistencia técnica especializada, la movilidad académica de los profesores capacitados, entre otros.

3. Los centros regionales de la UNAH deben considerar la figura de un profesor "integrador de tecnologías" que pueda ser capacitado constantemente en ciudad universitaria (Tegucigalpa), quien al regresar a su centro regional sea un agente constante de apoyo para el resto de los profesores de su área en la implementación de las TIC. Esta labor requiere que sea considerada como carga académica para asegurar su enfoque, ya que algunas TIC son muy especializadas, por lo que exigen la dedicación constante y la actualización. Lo anterior también implica reducir la movilidad de este profesor a otras áreas 0 asignaturas que signifiquen la pérdida de su labor como "integrador de tecnologías".

4. Las TIC implementadas en la asignatura Biología General II han contribuido al desarrollo de competencias conceptuales, procedimentales y actitudinales en los estudiantes de la Carrera de Biología.

5. El Proyecto Aulas Multimedia Autosuficientes ha cumplido el papel de una eficiente plataforma tecnológica-educativa en la Escuela de Biología.

6. El equipamiento multimedia permanente en las aulas puede ser considerado como una herramienta indispensable en la enseñanza de las ciencias biológicas. 


\section{BIBLIOGRAFÍA}

Canning, D. \& Cox, J. (2001). Teaching the structural nature of biological molecules: molecular visualization in the classroom and in the hands of students. Chemistry Education Research and Practice, 2, 109-122. DOI: 10.1039/B1RP90013G.

Eskicioglu, A. \& Kopec, D. (2003). The Ideal Multimedia-Enabled Classroom: Perspectives from Psychology, Education, and Information Science. Journal of Educational Multimedia and Hypermedia. 12 (2). Recuperado de: http://www.editlib.org/index.cfm?fuseaction=Reader.ViewAbstract\&paper_id=14575\&from= NEWDL\&a=1

García Ruiz, M.; Valdez Velázquez, L. y Gómez Sandoval, Z. (2008). Integración de visualización científica molecular en el salón de clases. Química Nova, 31(8), 2184-2189. Recuperado de: http://www.scielo.br/scielo.php?script=sci_arttext\&pid=S0100-40422008000800046\&lng=en\&tIng=pt.10.1590/S0100-40422008 000800046

Groot, M. D. (2002). Multimedia Projectors: A Key Component in the Classroom of the Future. THE Journal, 29(11), 18. Recuperado de: http://web.b.ebscohost.com/ehost/detail? sid=431ef07b-462c-49fb-bc8b-b56f23dcd64e\%40sessionmgr 114\&vid=1\&hid=117\&bdata=Jmxhbmc9ZXMmc2l0ZT1laG9zdC1saXZl\#db=bth\&AN=7670436

Laurillard, D. (1996).The Changing University. IT FORUM. Recuperado de: http:// www.researchgate.net/profile/Diana_Laurillard/publication/228938368_The _changing_university/links/0912f51156b52772eb000000.pdf

Muir Herzig, R. G. (2004). Technology and its impact in the classroom. Computers \& Education, 42(2), 111-131. Recuperado de: http://www.sciencedirect.com/science/article/pii/S0360131503000678

Muzyka, J. (2008). Visualization Tools for Organic Chemistry. Journal of Chemical Education, 86(2). Recuperado de: http://pubs.acs.org/doi/pdf/10.1021/ed086p25

Peat, M. \& Fernández, A. (2000). The role of information technology in biology education: an Australian perspective. Journal Of Biological Education (Society Of Biology), 34(2), 69. DOI:10.1080/00219266.2000.9655688

Pontes Pedrajas, A. (2005). Aplicaciones de las tecnologías de la información y de la comunición en la educación científica. Revista Eureka sobre Enseñanza y Divulgación de las Ciencias, 2(1), 2-18. Recuperado de: http://www.cienciamia.com.mx/fised/07tics/lecturas/aplicacion_tics2.pdf

Robinson, K. (2010). Changing education paradigms. RSA Animate. London: The Royal Society of Arts. Recuperado de: http://www.cfpscourseweb.com/pluginfi- 
le.php/1099/block_html/content/RSA\%20\%20Ken\%20Robinson\%20Lecture\% 20-\%20transcript.pdf

Stueker, O.; Brunberg, I.; Fels, G.; Borkent, H. \& Rooij, J. (2003). Web-Based Interactive Animation of Organic Reactions. Journal of Chemical Education, 80 (5). Recuperado de: http://pubs.acs.org/doi/pdf/10.1021/ed080p582

Thomas, J. \& Williamson, V. (2005). Molecular Visualization in Science Education: An Evaluation of the NSF-Sponsored Workshop. Journal of Chemical Education, 82 (6), 937. Recuperado de: http://pubs.acs.org/doi/abs/10.1021/ed082p937 Vicerrectoría Académica, UNAH. (2009). El Modelo Educativo de la UNAH. Tegucigalpa.

White, B.; Kim, S.; Sherman, K. \& Weber, N. (2002). Evaluation of molecular visualization software for teaching protein structure differing outcomes from lecture and lab: Differing outcomes from lecture and lab. Biochemistry and Molecular Biology Education, 30, 130-136. DOI: 10.1002/bmb.2002.494030020026

Williamson, V. \& Thomas, J. (2008). The Effects of a Two-Year Molecular Visualization Experience on Teachers' Attitudes, Content Knowledge, and Spatial Ability. Journal of Chemical Education, 85(5), 718-723. Recuperado de: http://eric.ed.go$\mathrm{v} /$ ?id=EJ826729

Zúniga, L. (2011). Innovación en el uso de tecnologías de información y comunicación en la UNAH: El caso de la Escuela de Biología. Informe técnico. Tegucigalpa: CEITICB-UNAH. Recuperado de: https://www.unah.edu.hn/?cat=4163\&fcats 